\title{
Opioid-galanin receptor heteromers differentiate the dopaminergic effects of morphine and methadone
}

\author{
Randal A. Serafini and Venetia Zachariou \\ Nash Family Department of Neuroscience and Department of Pharmacological Sciences, Icahn School of Medicine at Mount Sinai, New York, New York, USA.
}

\begin{abstract}
As the opioid addiction crisis reaches epidemic levels, the identification of opioid analgesics that lack abuse potential may provide a path to safer treatment of chronic pain. Preclinical studies have demonstrated that galanin affects physical dependence and rewarding actions associated with morphine. In the brain and periphery, galanin and opioids signal through their respective GPCRs, GaIR1-3 and the $\mu$-opioid receptor (MOR). In this issue of the $J C l$, Cai and collaborators reveal that heteromers between GaIR1 and MOR in the rat ventral tegmental area attenuate the potency of methadone, but not other opioids, in stimulating the dopamine release that produces euphoria. These studies help us understand why some synthetic opioids, such as methadone, do not trigger the release of dopamine in the mesolimbic system but still possess strong analgesic properties.
\end{abstract}

\section{Galanin system and} pharmacological responses to opioid alkaloids

A substantial number of patients with chronic pain develop physical dependence on prescription opioid painkillers and often become addicted. Opioid abuse-related deaths have reached an alarming level, with the NIH reporting over 50,000 US deaths in 2017 (1). Although the mechanisms by which synthetic opioids such as oxycodone and fentanyl exert their actions are partially understood, there are clear differences in the pharmacodynamic and pharmacokinetic properties of synthetic opioids used in clinical practice. All opioid painkillers are agonists of the $\mu$-opioid receptor (MOR), a GPCR that mediates the analgesic as well as euphoric effects of addictive opioid medications. In dopaminergic brain regions, specifically the ventral tegmental area (VTA), MOR activation potentiates dopamine release in the striatum and nucleus accumbens; this reward pathway is considered the anatomical crux of the addictive properties of opioids and other drugs of abuse. MOR is also the receptor for the synthetic opioid methadone, which is commonly used in rehabilitation programs because of its long half-life and its ability to prevent opiate withdrawal without promoting euphoric effects (2). To date, there is limited knowledge on the cellular actions that contribute to methadone's unique pharmacological profile.

The escalation of the opioid epidemic has galvanized interest in strategies that harness the analgesic power of opioids without their abuse potential. Over the past two decades, several preclinical studies documented the influence of the galanin system in behavioral responses to the opiate alkaloid morphine (3). Galanin, a multifunctional neuropeptide expressed in various cell types of the CNS, contributes to neuroendocrine function, learning and memory, mood, nociception, and appetite $(4,5)$. The diverse actions of galanin are mediated by three GPCR subtypes (GalR1, GalR2, and GalR3), which show unique

Related Article: p. 2730

Conflict of interest: The authors have declared that no conflict of interest exists.

distribution profiles in the brain and trigger distinct intracellular pathways (6). In rodent models, centrally administered galanin blocks the rewarding effects of morphine (7), whereas administration of galanin-like molecules attenuates symptoms of morphine withdrawal (8). Notably, in humans, variants in noncoding regions of $G A L$, the gene encoding galanin, have been identified as potential vulnerability factors for heroin addiction (9). Genomic studies in rodents have also identified changes in Gal expression upon morphine withdrawal (10), as well as an upregulation of Galr1 expression in the ventral striatum after chronic oxycodone self-administration (11). Previous work by Dr. Ferré's group identified heteromerization between MOR and GalR1 in the rat VTA and demonstrated that galanin disrupts opioid-induced dendritic dopamine release in rat VTA slices (12). This finding provided insight into the impact of the galanin system on GPCR dimerization, which is known to play a prominent role in functional responses to endogenous and synthetic opioids (13).

\section{Exploring the differences between methadone and morphine actions in the VTA}

In their recent study entitled "Opioidgalanin receptor heteromers mediate the dopaminergic effects of opioids," Cai et al. discover an agonist-dependent function of galanin-opioid receptor heteromers and provide insight into the mechanisms that control the rewarding potency of opioids (14). The study shows that MOR-GalR1 heteromers in the rat VTA are formed upon administration of methadone, ultimately inhibiting the release of dopamine in the VTA/nucleus accumbens pathway. In contrast, MOR-GalR1 heteromers did not alter the ability of morphine and fentanyl to stimulate dopamine release. This pharmacodynamic difference between methadone and highly addictive MOR agonists may provide a way to dissociate 
the euphoric versus therapeutic effects of methadone-like compounds. The findings of Cai et al. are supported by data showing substantially fewer self-reports of "high" sensations in patients on methadone maintenance compared with those using other opioids, confirming the lack of euphoric effects associated with methadone use. Thus, in addition to differences in brain penetrability, analgesic efficacy, ability to induce MOR internalization, recruitment of $\mathrm{G}$ protein complexes, and heteromerization with opioid receptor subtypes, MOR agonists may also differ in their ability to recruit heteromers containing galanin receptors.

\section{Future directions for studies on synthetic opioids}

These studies highlight not only the complex role of the galanin system in the neuroplasticity that drives physical dependence and addiction but also the need for a better understanding of the mechanisms mediating the actions of synthetic opioids beyond morphine and heroin. As the current opioid epidemic propels the identification of novel treatments for drug abuse and interventions that lower the addictive liability of opioid analgesics, understanding the actions of MOR-targeting opioids on MOR-galanin receptor heteromers may help define the potency of these opioids in activating the dopamine reward pathway. Future studies in models of drug addiction will further validate the opioid-galanin receptor heteromers as pharmacological targets and will help determine whether interventions targeting the function of these heteromers can provide a way to treat drug addiction or develop safer analgesic treatments. Given the diverse role of galanin receptors in essential physiological functions, it will also be important to understand the role of opioid-galanin receptor heteromers beyond the dopaminergic reward system.

Address correspondence to: Venetia Zachariou, 1425 Madison Ave, Box 10-65, New York, New York 10029, USA. Phone: 212.659.8612; Email: venetia.zachariou@ mssm.edu.

1. Scholl L, Seth P, Kariisa M, Wilson N, Baldwin G. Drug and opioid-involved overdose deaths United States, 2013-2017. MMWR Morb Mortal Wkly Rep. 2019;67(5152):1419-1427.

2. Novick DM, Salsitz EA, Joseph H, Kreek MJ. Methadone medical maintenance: an early 21st-century perspective. J Addict Dis. 2015;34(2-3):226-237.

3. Holmes A, Picciotto MR. Galanin: a novel therapeutic target for depression, anxiety disorders and drug addiction? CNS Neurol Disord Drug Targets. 2006;5(2):225-232.

4. Lang R, Gundlach AL, Kofler B. The galanin pep- tide family: receptor pharmacology, pleiotropic biological actions, and implications in health and disease. Pharmacol Ther. 2007;115(2):177-207.

5. Crawley JN. Biological actions of galanin. Regul Pept. 1995;59(1):1-16.

6. Freimann K, Kurrikoff K, Langel Ü. Galanin receptors as a potential target for neurological disease. Expert Opin Ther Targets. 2015;19(12):1665-1676.

7. Zachariou V, Parikh K, Picciotto MR. Centrally administered galanin blocks morphine place preference in the mouse. Brain Res. 1999;831 (1-2):33-42

8. Zachariou $\mathrm{V}$, et al. The neuropeptide galanin modulates behavioral and neurochemical signs of opiate withdrawal. Proc Natl Acad Sci U S A. 2003;100(15):9028-9033.

9. Levran O, et al. Genetic susceptibility to heroin addiction: a candidate gene association study. Genes Brain Behav. 2008;7(7):720-729.

10. McClung CA, Nestler EJ, Zachariou V. Regulation of gene expression by chronic morphine and morphine withdrawal in the locus ceruleus and ventral tegmental area. J Neurosci. 2005;25(25):6005-6015.

11. Zhang Y, Liang Y, Randesi M, Yuferov V, Zhao C, Kreek MJ. Chronic oxycodone self-administration altered reward-related genes in the ventral and dorsal striatum of C57BL/6J mice: an RNA-seq analysis. Neuroscience. 2018;393:333-349.

12. Moreno E, et al. Functional $\mu$-opioid-galanin receptor heteromers in the ventral tegmental area. J Neurosci. 2017;37(5):1176-1186.

13. Jordan BA, Devi LA. G-protein-coupled receptor heterodimerization modulates receptor function. Nature. 1999;399(6737):697-700.

14. Cai NS, et al. Opioid-galanin receptor heteromers mediate the dopaminergic effects of opioids. JClin Invest. 2019:129(7):2730-2744. 